\title{
Performance Study of COPS over TLS and IPsec Secure Session
}

\author{
Yijun Zeng and Omar Cherkaoui \\ Université du Québec à Montréal \\ CP. 8888 Suc. A, Montréal \\ Yj_zeng@hotmail.com, cherkaoui.omar@uqam.ca
}

\begin{abstract}
This paper evaluates the performance of COPS over secure TLS and IPsec connections. For large size data, when we apply authentication and encryption, the throughput degrades compared with the throughput without authentication or encryption. COPS has native security mechanisms, but it also has limitations. As defined in RFC 2478, COPS includes no standard key management and no data privacy hop-by-hop security. To be deployed, it needs to support access control models. Based on our comparison of the performance of the implementation of COPS, COPS over TLS and COPS over IPsec, we propose a strategic approach to secure COPS.
\end{abstract}

Keywords: COPS, IPsec, and TLS

\section{Introduction}

COPS [1] was designed to distribute cleartext policy information from a centralized Policy Decision Point (PDP) to a set of Policy Enforcement Points (PEP) over the Internet. COPS provides its own security mechanisms to protect the per-hop integrity of the deployed policy.

However, the use of COPS for sensitive applications such as some types of security policy distribution requires additional security measures, such as data privacy. This is because some organizations need to hide some or all of their security policies, e.g., because policy distribution to devices such as mobile platforms can cross domainboundaries.

COPS can reuse existing protocols for security such as IPsec [4] or TLS [2] to authenticate and secure the channel between the PDP and PEPs. TLS was designed to provide channel-oriented security. It provides mechanisms for both one- and two-way authentication, dynamic session keying, and data stream privacy and integrity. IPsec provides security services similar to those of TLS. There are also differences when using these two protocols to secure COPS.

As is well known, the required processing for security functions is not light, and tends rather to be large. When the execution of security function (TLS or IPsec) requires very large processing power, we may lack throughput for many applications. 
Or else, we may need to implement special hardware to handle those security functions.

This paper evaluates the complexity of the implementation of Pure COPS, COPS over TLS and COPS over IPsec.

\section{Three Scenarios for Implementing COPS}

We designed three scenarios to study COPS performance with and without security features: Pure COPS, COPS over TLS and COPS over IPsec. The three scenarios in the protocol stack are shown in Fig. 2.1. This section will give a brief description of the three scenarios.

Pure COPS

\begin{tabular}{|c|}
\hline COPS \\
\hline TCP \\
\hline IP \\
\hline
\end{tabular}

COPS over TLS

\begin{tabular}{|c|}
\hline COPS \\
\hline TLS \\
\hline TCP \\
\hline IP \\
\hline
\end{tabular}

COPS over IPsec

\begin{tabular}{|c|}
\hline COPS \\
\hline TCP \\
\hline IPsec \\
\hline IP \\
\hline
\end{tabular}

Fig. 2.1. Three scenarios for COPS implementation

\subsection{Pure COPS}

COPS is a standards-track protocol specification from IETF designed for communicating policy information within a policy management system. It is a clientserver communication model that includes acknowledgement sent upon the successful processing of messages. The COPS server resides at the PDP that responds to the request within the COPS clients at PEPs. COPS uses TCP as its transport protocol for reliable exchange of messages between policy clients and a server. When implementing COPS without additional security features, we call such a scenario Pure COPS.

The basics operations are Client-Open (OPN), Client-Close (CC), Client-Accept (CAT), Keep-Alive (KA), Request (REQ), Decision (DEC), Report State (RP), Delete Request State (DRQ), Synchronize State Request (SSQ) and Synchronize Complete (SSC). These messages contain command codes that instruct various operations on client-specific PIB entities. An object Integrity will be in the messages when COPS' native security feature is used. The contents of Integrity are a 96-bit MD5 HMAC computed on the message contents and a sequence number. But all policy information is distributed in cleartext. 


\subsection{COPS over TLS}

The TLS protocol was designed to establish a secure connection between two communication peers. To establish such a secure connection, TLS performs a handshake between these two peers to authenticate them and agree upon a set of security parameters. The negotiated security parameters are used for calculating a shared session $H D R, S A$ ect the data transmission between these two peers.

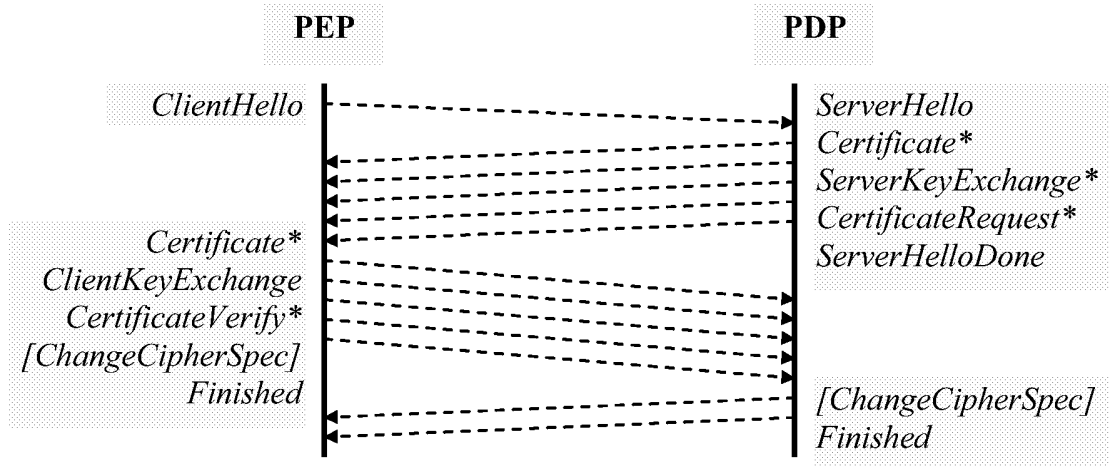

* Indicates optional messages that are not always sent.

Fig. 2.2. A Complete TLS Handshake between the PDP and PEP in COPS/TLS

The implementation of COPS over TLS is described in the IETF Internet Draft "COPS Over TLS" [3]. We abbreviate COPS over TLS to COPS/TLS in the rest of this paper. When implementing COPS/TLS, TLS resides on top of TCP and underneath COPS in the protocol stack (Fig. 2.1). A COPS/TLS session begins with a TLS handshake between the PDP and PEP. The PDP and PEP are responsible for establishing the TLS connection before any COPS messages can be exchanged. The PDP and PEP can exchange the first COPS message OPN and CAT only after the TLS connection is established. All COPS messages are exchanged in ciphertext.

\subsection{COPS over IPsec}

RFC 2401 [4] describes the architecture framework of IPsec. The IPsec protocol suite provides a functional suite for secure and reliable data exchange over the Internet. IPsec has the following two functions: Authentication and Encryption. IPsec defines AH (Authentication Header, RFC 2402 [5]) for user validation of the communication peer and the ESP (Encapsulating Security Payload, RFC2403 [6]) for encrypting and encapsulating user data in IP packets. Fig. 2.3 indicates the messages exchanged when establishing an IPsec connection. 


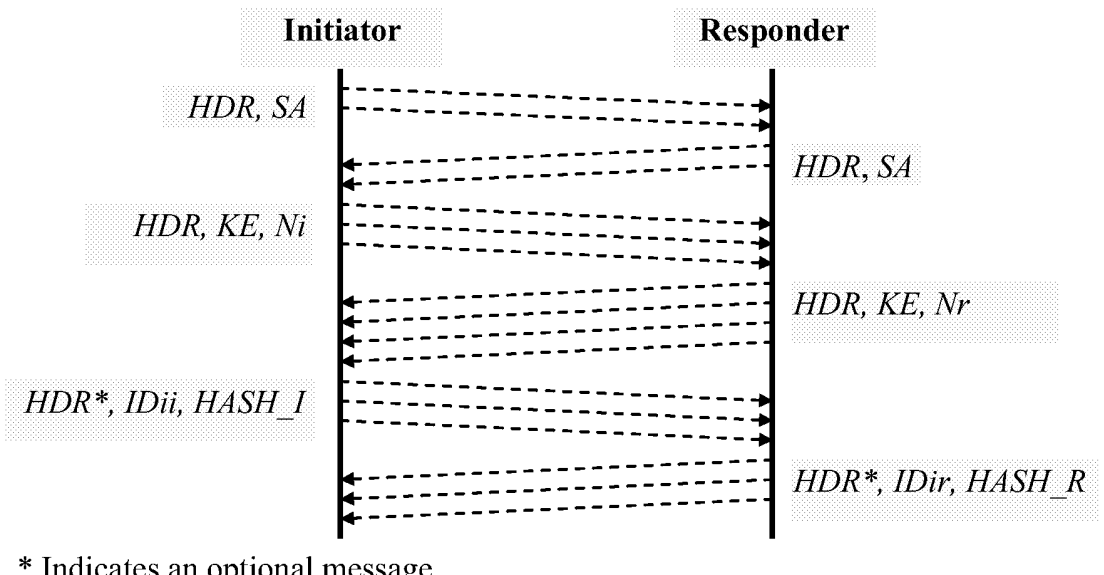

* Indicates an optional message

Fig. 2.3. Phase 1 Authentication with a Pre-shared Key (Main Mode)

When running COPS over IPsec, COPS and IPsec are not adjacent in the protocol stack (Fig. 2.1). Therefore running COPS over IPsec is quite simple. A secure IPsec connection is established between the two hosts where the PDP and PEP reside at respectively. It protects the COPS messages exchanged between the PDP and PEP by protecting all TCP traffic between these two hosts. The PDP and PEP are not responsible for establishing the IPsec connection. All the jobs that the PDP and PEP do over IPsec are exactly the same as in Pure COPS after the IPsec connection established. We abbreviate COPS over IPsec to COPS/IPsec in the rest of this paper.

Table 2.1. Security Algorithms used in TLS and IPsec

\begin{tabular}{|c|c|c|}
\hline Supported Services & TLS & IPsec \\
\hline Peers Authentication & X.509 Certificate & $\begin{array}{c}\text { X.509 Certificate } \\
\text { Pre-shared Key } \\
\text { Kerberos }\end{array}$ \\
\hline Key Exchange & RSA, Diffie-Hellman & RSA, Diffie-Hellman \\
\hline Encryption Algorithms & DES, 3DES, RC4 & DES, 3DES \\
\hline MAC Algorithms & MD5, SHA-1 & MD5, SHA-1 \\
\hline
\end{tabular}




\subsection{TLS vs. IPsec}

TLS and IPsec are in different layer in the protocol stack, but they provide similar security services by using similar security algorithms. Table 2.1 gives a view of the algorithms that TLS and IPsec support respectively.

\subsection{The Implementations}

We developed an application SecureCops in Java to simulate the three scenarios described previously. SecureCops integrates TLS security services into COPS. It consists of two components: PDPSimulator and PEPSimulator which act as a PDP and PEP respectively. The TLS module for COPS/TLS was developed with our Java API library JTLS. When simulating Pure COPS, SecureCops performs COPS operations complying with RFC 2748.

When simulating COPS/TLS, A TLS handshake will be performed between PDPSimulator and PEPSimulator before any COPS operations can be carried out. The TLS module complies with RFC 2246 and COPS/TLS complies with the IETF Internet Draft "COPS Over TLS" [3]. The COPS operations over TLS are exactly the same as it simulates Pure COPS, but the messages are redirected to TLS, not to TCP.

When simulating COPS/IPsec, SecureCops does not touch any IPsec library since COPS and IPsec are not adjacent in the protocol stack. PDPSimulator and PEPSimulator exchange COPS messages by sending messages to and receiving messages from TCP without any awareness that IPsec encrypts and decrypts the sending and receiving messages. SecureCops uses the existing IPsec implementation in the operating system to get security services.

\section{Testbed Configuration}

Our simulations were done on Windows 2000 on normal PCs.

\subsection{System Environment}

We run SecureCops on two PCs, both with an Intel Pentium III $700 \mathrm{~Hz}$ processor, 128 MB memory and the Windows 2000 Professional operating system, the PDP PDPSimulator running on one PC and the PEP PEPSimulator on the other. These two PCs are connected with a hub (ASANTÉ FriendlyNet, 8-Port Ethernet). Since SecureCops is programmed in Java, the Java Platform on both PCs is Java(TM) 2 Runtime Environment, Standard Edition (build 1.4.0-b92).

\subsection{Security Configuration}

For COPS/TLS, security parameters should be configured on SecureCops. We set the TLS cipher suite to $T L S \_R S A \_W I T H \_3 D E S \_E D E \_C B C \_S H A$. Therefore it uses RSA 
for key exchange, 3DES for encryption and SHA-1 for MAC. A self-signed X.509 certificate is used to authenticate the PDP and PEP convey the server's public key.

For COPS/IPsec, since we use the IPsec implementation built in Windows 2000 Professional, the configuration should be done on the operating system. In the Windows 2000 configuration, we set the Authentication Method to the Pre-shared Key authentication, IP Security Policy to Secure Server (Require Security) and Security Methods to 3DES for ESP confidentiality and SHA-1 for ESP integrity.

\section{Results and Analysis}

To study the performance of the three scenarios of COPS implementation, we divide a COPS session into two parts: the connection procedure and the COPS operations. Our performance study is based on the timing of these two parts: the time for connection and the time for the operations. We run SecureCops to simulate the three scenarios. For each of these three scenarios, we repeated the simulation five times and recorded the time for connection, and the time for operations at the PDP (PDPSimulator) side and PEP (PEPSimulator) side.

For simplification, the simulation of a session in each of the three scenarios exchanges five COPS messages: OPN, CAT, REQ, DEC and CC. The two parts of a session in the simulations and the time we recorded are shown in Fig. 4.1.

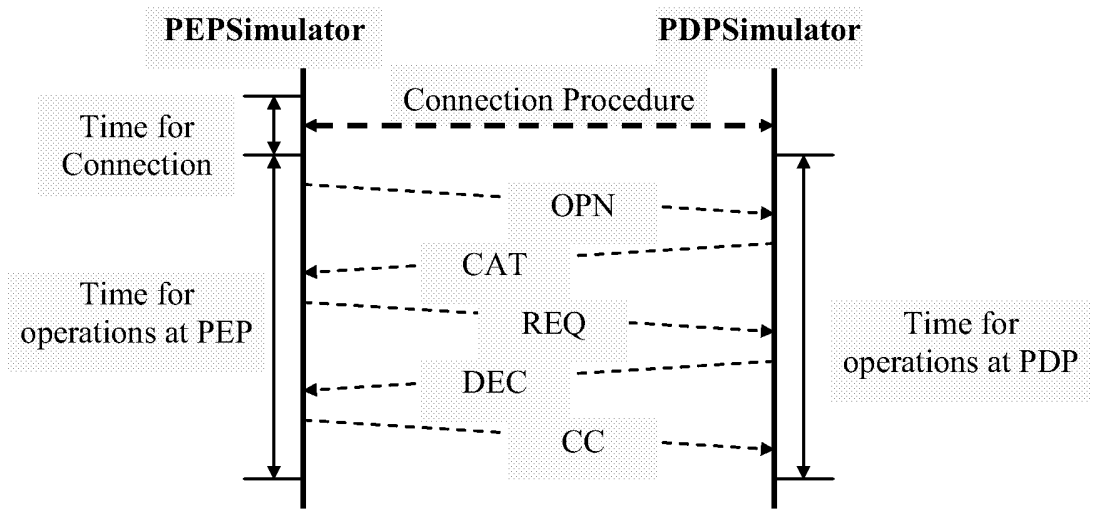

Fig. 4.1. Simulation of a session in each of three scenarios

\subsection{Time for Connection}

The time we recorded for establishing a COPS connection between PDPSimulator and PEPSimulator is the execution time of the constructor method Socket from the java.net.Socket class in both Pure COPS and COPS/IPsec, and the execution time of 
the constructor method TLSSocket and the method clientHandshake from the class JTLS.Connection.TLSSocket in COPS/TLS. The connection time for five simulations in the three scenarios is shown in Table 4.1.

Table 4.1. Time for connection (millisecond)

\begin{tabular}{|c|c|c|c|c|c||c|}
\hline Scenario & Simu. 1 & Simu. 2 & Simu.3 & Simu.4 & Simu.5 & Ave. \\
\hline Pure COPS & 20 & 30 & 20 & 20 & 30 & 24 \\
\hline COPS/TLS & 23985 & 23984 & 24115 & 23995 & 24095 & 24034.8 \\
\hline COPS/IPsec & 851 & 644 & 571 & 571 & 650 & 657.4 \\
\hline
\end{tabular}

Among these three scenarios, Pure COPS took the shortest time for the connection. The average connection time in the five simulations is only $24 \mathrm{~ms}$, because it did not carry out any additional operations, just simply establishing a TCP connection.

COPS/IPsec took much longer for the connection. Its average connection time in the five simulations is 27.39 times that with Pure COPS. During the connection (execution of the method Socket), IPsec performed a security negotiation between the two PCs where PDPSimulator and PEPSimulator were running. Therefore extra time is needed to generate and exchange those messages, and to perform security computation.

COPS/TLS took the longest time for connection. Its average connection time is 1001.45 times as much as of Pure COPS and 36.56 times as much as of COPS/IPsec.. After establishing a TCP connection between PDPSimulator and PEPSimulator, the two parties have to perform the TLS handshake described in Fig. 2.2. They exchange all handshake messages except for ServerKeyExchange, because they exchange the session key with RSA and the server's public key has been conveyed in the server's X.509 certificate.

During the handshake in COPS/TLS, the generation of three messages ClientHello, ServerHello and ClientKeyExchange consumed the most connection time. For the first two messages, each needs a 28-byte random number and the last needs a 46-byte random number. Random number generating is a time consuming computation. Fig. 4.2 shows the time for generating those three messages in each of five simulations. In each simulation, generating these three messages consumed at least $85 \%$ of the connection time. 


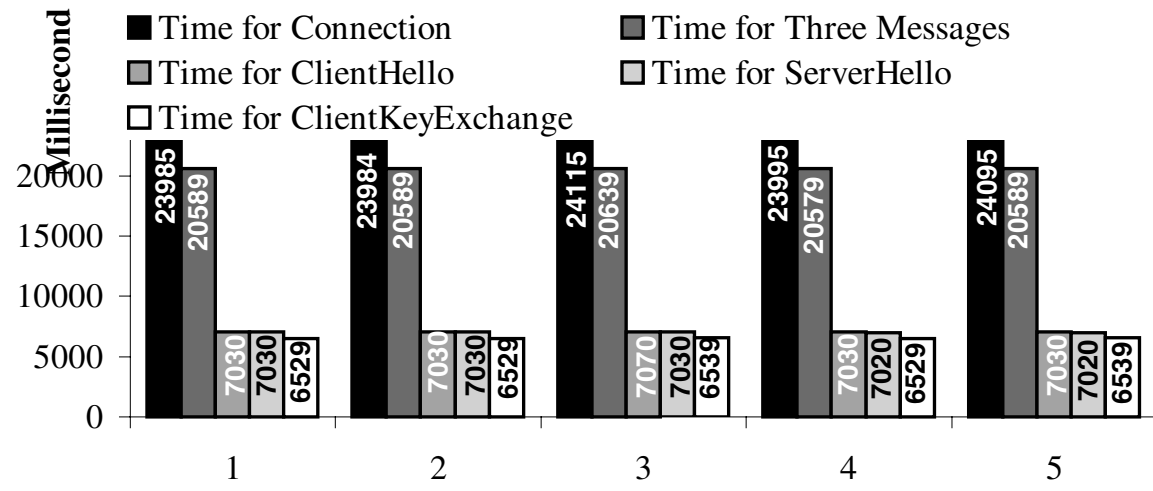

Simulation

Fig. 4.2. Time for generating the three handshake messages

\subsection{Time for PEP to Complete the COPS Operations}

In our simulations of all three scenarios, the COPS operations in a session include five COPS message exchanges, which are shown in Fig. 4.1. The time for the PEP to complete the COPS operations in a session we recorded is the interval from the point PEPSimulator begins to send the message OPN until it finishes sending the last message CC and shuts down the connection, as indicated in Fig. 4.1. The time for the PEP to complete the COPS operations in a session for all three scenarios in all five simulations is shown in Fig. 4.3. For example, in the simulation 1, PEPSimulator took $410 \mathrm{~ms}$ to complete the COPS operations for Pure COPS, $450 \mathrm{~ms}$ for COPS/TLS and $521 \mathrm{~ms}$ for COPS/IPsec.

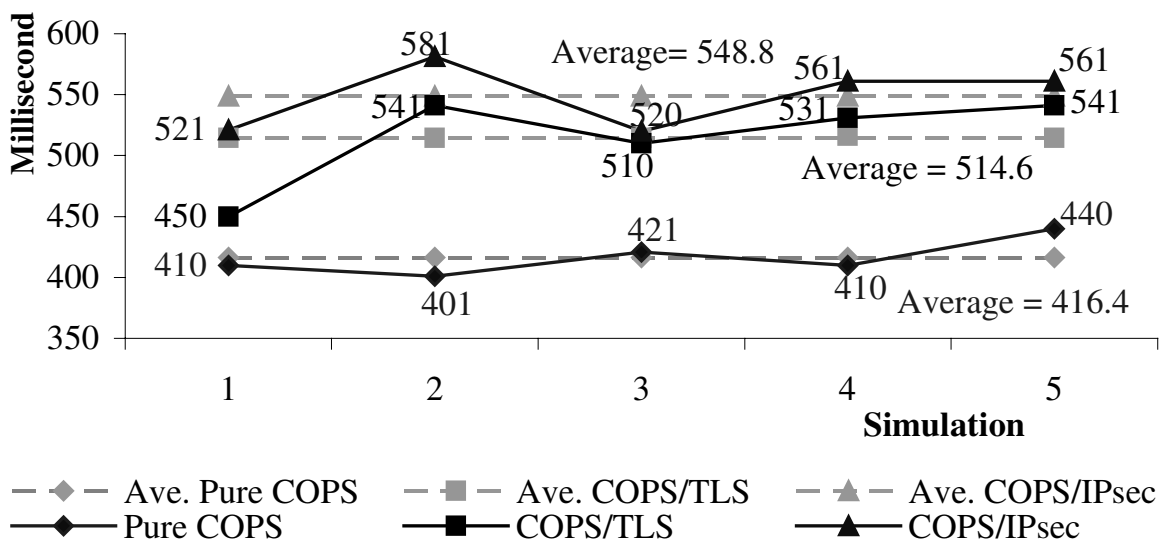

Fig. 4.3. Time for PEP to complete a whole session for three scenarios in all five simulations 
In all five simulations, Pure COPS always took shortest time to complete the COPS operations; the average is $416.4 \mathrm{~ms}$. In Pure COPS, the PDP and PEP just put the message down to TCP when sending or get the received message from TCP when receiving.

In COPS/TLS, TLS encrypts the messages to be sent before they can be handled down to TCP, and decrypts the received messages after taking them from TCP but before delivering to the receiving PDP or PEP. In COPS/IPsec, IPsec encrypts the messages to be sent after the PDP or PEP sends them down to TCP, and when the PDP or PEP gets the received messages from TCP, the messages have already been well decrypted by IPsec. Therefore extra time was taken to complete the COPS operations in these two secure COPS scenarios.

On average for the five simulations, COPS/TLS took $514.6 \mathrm{~ms}$ to complete the COPS operations in a COPS session, 1.24 times as much as Pure COPS, and COPS/IPsec took $548.8 \mathrm{~ms}, 1.34$ times as much as Pure COPS. The difference between the time for the secure COPS session and the Pure COPS session is not so big as that for establishing a connection.

\subsection{Time for PDP to Complete the COPS Operations}

Once the connection is established, the PDP is ready to receive the message OPN from the PEP, and will shut down the connection to the PEP after receiving the message CC from the PEP. The time for the PDP to complete the COPS operations in a session we recorded is the interval from the point PDPSimulator gets ready to receive the message OPN until it finishes receiving the message $\mathrm{CC}$ and shuts down the connection to PEPSimulator, as indicated in Fig. 4.1.

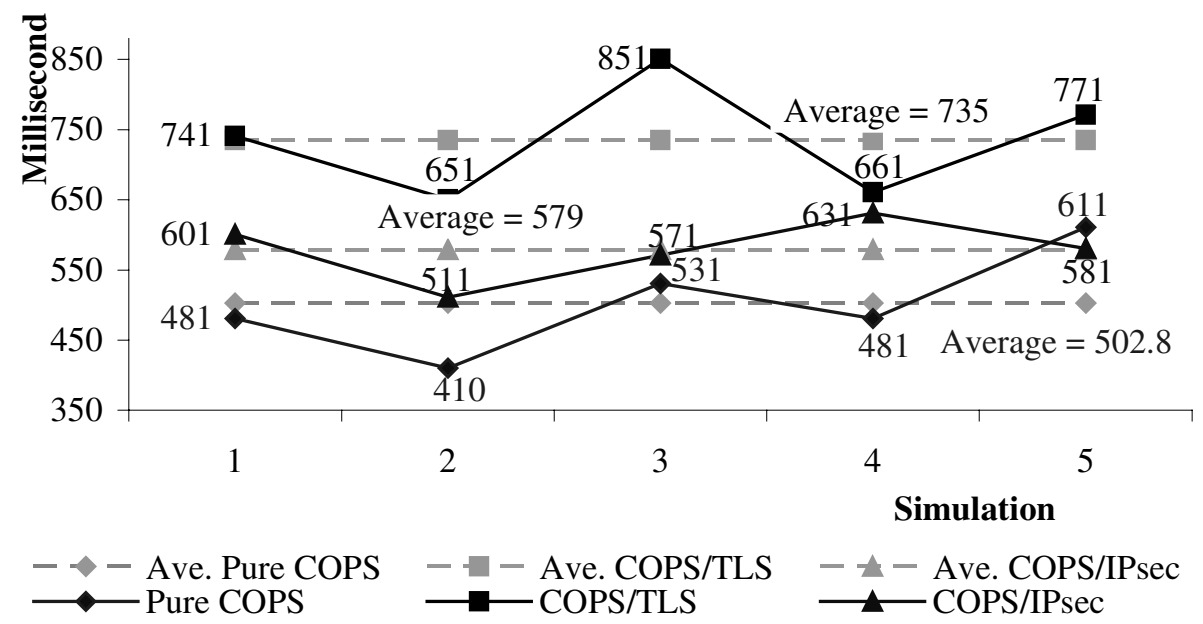

Fig. 4.4. Time for PDP to complete a whole session for three scenarios in all five simulations 
On the PDP side, Pure COPS session once again took the shortest time to complete the COPS operations, because it does not perform encryption and decryption when sending and receiving. On average for the five simulations, PDPSimulator took 502.8 ms to complete the session in Pure COPS, $579 \mathrm{~ms}$ in COPS/IPsec and $735 \mathrm{~ms}$ in COPS/TLS. This average time for COPS/IPsec is 1.15 times that of Pure COPS, and in COPS/TLS it is 1.46 times that of Pure COPS.

We found that PDPSimulator always took longer than PEPSimulator did to complete the COPS operations for a given scenario. Let us take a look again at Fig. 4.1. In a session of our simulations, PDPSimulator receives three messages (OPN, REQ and CC) and sends two (CAT and DEC). By contrast, PEPSimulator sends three messages (OPN, REQ and CC) and receives two (CAT and DEC).

PDPSimulator or PEPSimulator sends messages by calling the method write from the class java.io.DataOutputStream in Pure COPS and COPS/IPsec, and calling the method tlsWrite from the class JTLS.TLSio.TLSOutputStream in COPS/TLS, and receives messages by calling the method read from the class java.io.DataInputStream in Pure COPS and COPS/IPsec, and calling the method tlsRead from the class JTLS.TLSio.TLSInputStream in COPS/TLS.

write or tlsWrite returns immediately when the execution finishes. However, read or tlsRead blocks until the input data is available. When PDPSimulator or PEPSimulator calls read or tlsRead to receive messages, the sender may still prepare and generate the messages to be sent while PDPSimulator or PEPSimulator is waiting for the data available. Therefore message-receiving takes longer than messagesending does. In a session of our simulation, PDPSimulator receives one more message than PEPSimulator does. This is why PDPSimulator took longer than PEPSimulator does to complete the COPS operations in a session.

\subsection{Security Overhead and COPS Operations}

No matter in which of our three scenarios, COPS always handles the data in cleartext. The time for generating and verifying Integrity we recorded is always zero millisecond, e.g. it is too short for the PCs to record. Therefore we can say the COPS operations do not cause any security overhead.

\section{Conclusion}

The simulations of three scenarios, Pure COPS, COPS/TLS and COPS/IPsec, showed that 3DES encryption and SHA-1 do affect the COPS performance when sending and receiving COPS messages, but the difference is not significant. Both TLS and IPsec provide strong security services to protect the COPS policy with confidentiality and integrity. 


\subsection{Performance in Time Consumption}

As we saw from the comparison between the three scenarios, exchanging COPS messages in ciphertext does not increase time by much as compared with exchanging the messages in cleartext. In both COPS/TLS and COPS/IPsec, the encryption algorithms do not cause significant delay in COPS message exchange. Therefore paying a little price in time consumption can ensure confidentiality protection for COPS policy information distribution.

But when establishing the connection between the PDPSimulator and PEPSimulator, the time cost tells a different story. Time is the price to pay to establish a security connection between a COPS PDP and PEP. To establish a security connection over TLS between PDPSimulator and PEPSimulator, it took about 24 seconds, 1001.45 times as much as needed for a Pure COPS connection, about 0.6 seconds for a connection over IPsec, 36.56 times as much as for a Pure COPS.

To add security feature to the COPS protocol, with TLS or with IPsec, it is better to make the security session reusable. When establishing a security connection between a PDP and PEP, using the security parameters negotiated by a previous session can greatly reduce the connection time by avoiding the time consuming computations, for example, the random numbers generation in the TLS handshake, during a complete security negotiation. At the same time, a policy should be established to regulate how long and how many times a set of negotiated security parameters can be reused.

\subsection{Deployment of COPS/IPsec}

Running COPS/IPsec is quite simple. Since COPS and IPsec are not adjacent in the protocol stack, the implementation does not need to touch any IPsec API library. No modification is needed in a COPS implementation in order to use an existing IPsec implementation. Just configuring the existing IPsec in the operating system, IPsec will protect the COPS policy information exchanged between a PDP and PEP by protecting all TCP traffic between these two hosts where the PDP and PEP reside at respectively.

The disadvantage of using IPsec is that IPsec cannot provide a COPS oriented access control when establishing the security connection. Therefore, for COPS there is no authentication of the COPS client to the COPS server (since there is no mapping between the IPsec identity and the PEPID) during the security negotiation. IPsec protects the COPS policy information by protecting all TCP traffic between the two hosts. This means that if there are other applications communicating between these two hosts, they will use the same IPsec session.

If there is any Network Address Translation (NAT) between the two endpoints, IPsec does not work. In this case, COPS cannot get security services from IPsec. IPsec needs more system configurations. 


\subsection{Deployment of COPS/TLS}

Since TLS is adjacent to COPS in the protocol stack, it is possible to establish a COPS oriented security connection by running COPS/TLS. If there are other applications communicating between the same hosts the PDP and PEP reside at and those applications have no sensitive information to be protected, it seems that IPsec does too much considering that encryption algorithms require very large processing power when computing on a large data block. However, the use of COPS/TLS gives COPS a session oriented secure connection while other applications can still exchange messages in cleartext if they do not want to hide their information or the messages are not sensitive.

If other applications want their conversation to be secured, they can establish their own security connection by using TLS. Therefore, a security session for COPS is separated from any other security sessions for other applications, and it is possible to provide the authentication of the COPS client to the COPS server. The security configuration for using TLS is only done in the COPS application. There is no NAT problem when using COPS over TLS.

The disadvantage is that COPS applications should be modified to support TLS. This modification will be much work for the developers.

\section{References}

1. D. Durham, J. Boyle, R. Cohen, S. Herzog, R. Rajan and A. Sastry, "The COPS (Common Open Policy Service) Protocol", IETF RFC 2748, January 2000

2. T. Dierks and C. Allen, "TLS Protocol version 1.0", IETF RFC 2246, January 1999

3. Jesse Walker and Amol Kulkarni, "COPS Over TLS", IETF Internet Draft, June 2002, http://www.ietf.org/internet-drafts/draft-ietf-rap-cops-tls-04.txt

4. S. Kent and R. Atkinson, "Security Architecture for the Internet Protocol", IETF RFC 2401, November 1998.

5. S. Kent and R. Atkinson, "IP Authentication Header", IETF RFC 2402, November 1998.

6. S. Kent and R. Atkinson, "IP Encapsulation Security Payload (ESP)", IETF RFC 2403, November 1998.

7. D. Harkins and D. Carrel, "The Internet Key Exchange (IKE)", IETF RFC 2409, November 1998.

8. S. Deering and R. Hinden, "Internet Protocol version 6 Specification", IETF RFC 2460, November 1998.

9. H. Orman, "The OAKLEY Key Determination Protocol", IETF RFC 2412, November 1998.

10. J. Postel, "Internet Protocol", IETF RFC 791, September 1981.

11. J. Walker, "CMS Over COPS", IETF Internet Draft, May 2000. http://www.alternic.org/drafts/drafts-j-k/draft-jwalker-cops-cms-00.txt 